\title{
Presynaptic Calcium/Calmodulin-Dependent Protein Kinase II Regulates Habituation of a Simple Reflex in Adult Drosophila
}

\author{
Ping Jin, ${ }^{1}$ Leslie C. Griffith, ${ }^{2}$ and R. K. Murphey ${ }^{1}$ \\ ${ }^{1}$ Department of Biology, Neuroscience and Behavior Program, University of Massachusetts, Amherst, Massachusetts \\ 01003, and 2Biology Department and the Volen Center for Complex Systems, Brandeis University, Waltham, \\ Massachusetts 02254
}

\begin{abstract}
On repetitive stimulation, the strength of a reflex controlling leg position in Drosophila decreased, and this response decrement conformed to the parametric features of habituation. To study the presynaptic function of CaMKII in this nonassociative form of learning, we used a P[Gal4] insertion line to target the expression of mutant forms of CaMKII to the sensory neurons controlling the reflex. Targeted expression of a calciumindependent CaMKII construct (T287D) in the sensory neurons eliminated habituation. Targeted expression of a mutant CaMKII incapable of achieving calcium independence (T287A) reduced
\end{abstract}

The study of the molecular basis of learning and memory is focused on a variety of biochemical pathways and second messenger systems. One that is strongly implicated as a molecular substrate for learning and memory is CaMKII (Silva et al., 1992a,b; Griffith et al., 1993; Mayford et al., 1995, 1996; Giese et al., 1998). On activation by calcium/calmodulin, CaMKII can phosphorylate a variety of substrates, including itself (for review, see Braun and Schulman, 1995), and this autophosphorylation converts the kinase to a calcium-independent protein kinase for which the activity can last much longer than the original calcium signal (Hanson et al., 1989; Hanson and Schulman, 1992). This switch from calcium dependence to calcium independence and the rather long survival of the calcium-independent form suggest the hypothesis that CaMKII is a molecular memory device (Miller and Kennedy, 1986; Lisman and Goldring, 1988).

Mutational studies of CaMKII function in both mice and flies supported the idea that this molecule was crucial to learning. Knock-out mice, lacking CaMKII, exhibited impaired spatial learning (Silva et al., 1992a) and hippocampal long-term potentiation (LTP) (Silva et al., 1992b). Similarly, mutation of the CaMKII gene or inhibition of CaMKII by expressing a peptide that blocked kinase function in Drosophila impaired performance in a courtship conditioning test and altered larval neuromuscular transmission (Griffith et al., 1993; Wang et al., 1994; Joiner and Griffith, 1997).

\footnotetext{
Received May 19, 1998; revised July 14, 1998; accepted Aug. 14, 1998.

This work was supported by National Science Foundation Grants IBN 95-14701 to R.K.M. and IBN 94-21360 to L.C.G. We thank Drs. Randall Phillis and James R. Trimarchi for help and discussion during this study. Dr. Carol Bigelow and Susanne May provided advice and help with the statistical analysis. Suman Reddy, Phyllis Caruccio, and Michael Getzinger screened the P[Gal4] insertion lines and provided expert anatomical assistance throughout the project. Dr. John Lisman kindly provided helpful comments on an early draft of this manuscript.

Correspondence should be addressed to Dr. R. K. Murphey, Department of Biology, Morrill Science Center, University of Massachusetts, Amherst, MA 01003.

Dr. Jin's present address: Biochemistry Department and Volen Center for Complex Systems, Brandeis University, Waltham, MA 02254.

Copyright (C) 1998 Society for Neuroscience $\quad 0270-6474 / 98 / 188955-10 \$ 05.00 / 0$
}

the initial reflex response, but a strong facilitation then occurred, and this eliminated most of the habituation. Finally, when a CaMKII inhibitory peptide (ala) was expressed in sensory neurons, the initial response was reduced, followed by facilitation. These results suggest that basal CaMKII levels in the presynaptic neurons set the response level and dynamics of the entire neural circuit.

Key words: CaMKII; habituation; presynaptic; Drosophila; reflex; $P$ [Gal4]

The relative role of CaMKII in pre- versus postsynaptic cells is of great interest, and a variety of studies is concerned with determining the locus of CaMKII function. Strong evidence points to the postsynaptic neurons in the hippocampus, where postsynaptic injection of a CaMKII inhibitor blocks the induction of LTP (Malinow et al., 1989). Other results demonstrate that one of the substrates of CaMKII, the AMPA receptor, is phosphorylated after LTP induction and that the phosphorylation can last as long as $1 \mathrm{hr}$ (Barria et al., 1997). Finally, genetic manipulation of CaMKII in the postsynaptic neurons demonstrates a postsynaptic function of CaMKII in learning (Mayford et al., 1996). Thus, much of the work on the hippocampus argues for a postsynaptic site of action for CaMKII and direct modification of postsynaptic proteins in producing LTP.

In contrast to the strong evidence for a postsynaptic role, the presynaptic role of CaMKII in the regulation of synaptic plasticity is less well understood. Early studies in which CaMKII was injected presynaptically into the squid giant synapse demonstrated that transmitter release was increased, possibly via the phosphorylation of synapsins (Llinás et al., 1985). However, synaptic plasticity was not explored in this preparation. To examine the role of CaMKII in presynaptic plasticity, we have examined habituation in the thoracic nervous system of Drosophila. Habituation in Drosophila has been studied in a number of behavioral and electrophysiological preparations, including the proboscis extension reflex (Duerr and Quinn, 1982), the landing response (Asztalos et al., 1993), and the visual escape jump (Engel and Wu, 1996). However, in each of these cases the neural circuit underlying the behaviors is relatively complex, and it is difficult to analyze the specific synapses underlying the behavioral plasticity. For example, habituation of the visually evoked escape jump response involves one of the best known neural circuits in Drosophila, in which the circuitry postsynaptic to the giant interneuron is well characterized and each cell and each synapse in the circuit is uniquely identified. However, the synaptic modification 


\begin{tabular}{|c|c|c|c|c|c|c|}
\hline & First peak & $\begin{array}{l}\text { Comparison of } \\
\text { first peaks }\end{array}$ & Slope & $\begin{array}{l}\text { Test for nonzero } \\
\text { slope }^{c}\end{array}$ & $\begin{array}{l}\text { Paired com- } \\
\text { parison }^{b}\end{array}$ & $n^{d}$ \\
\hline 287D & $191+11.8$ & & 0.00028 & $\mathrm{p}=0.52$ & & 7 \\
\hline Control $^{e}$ & $184+10.6$ & $p=0.67$ & 0.0088 & $\mathrm{p}<0.0001$ & $p<0.0001$ & 7 \\
\hline 287D stong & $165+5.5$ & & 0.00038 & $\mathrm{p}=0.32$ & & 5 \\
\hline 287D weak & $110+8.5$ & $p<0.0001$ & 0.0016 & $\mathrm{p}=0.09$ & $p<0.0001$ & 5 \\
\hline $287 \mathrm{~A}$ & $212+11.4$ & & 0.0019 & $\mathrm{p}=0.05$ & & 7 \\
\hline Control $^{e}$ & $148+6.8$ & $p<0.0001$ & 0.0083 & $\mathrm{p}<0.0001$ & $p<0.0001$ & 7 \\
\hline ala & $119+6.8$ & & 0.000063 & $\mathrm{p}=0.94$ & & 7 \\
\hline Control $^{e}$ & $214+6.9$ & $p<0.0001$ & 0.011 & $\mathrm{p}<0.0001$ & $p<0.0001$ & 7 \\
\hline
\end{tabular}

The formula for exponential decay: $y=a \exp [-b t]$, where $a$ is the intercept and $b$ the slope.

${ }^{a}$ Repeated measures $A N O V A$.

${ }^{b}$ Wald $\chi^{2}$ statistic with two degrees of freedom.

${ }^{c}$ Wald test of the null hypothesis that the rate of decay is zero (two-tailed values).

${ }^{d}$ Each specimen was tested with 10 series of 15 flexion movements at $2 \mathrm{~Hz}$.

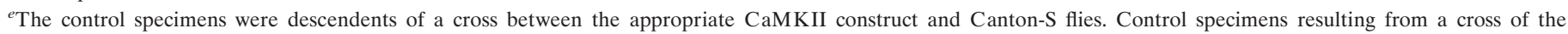
$\mathrm{P}[\mathrm{Gal} 4]-\mathrm{c} 362$ line and CS gave similar results.

during habituation of the escape response apparently occurs presynaptic to the giant fiber, and the relevant synapses therefore are effectively hidden from the electrophysiologist (Engel and $\mathrm{Wu}, 1996)$. To overcome the difficulties associated with the complexity of these neural circuits and study specific synapses, we identified a simple proprioceptive reflex controlling leg position in adult flies. We used a $\mathrm{P}[\mathrm{Gal} 4]$ enhancer trap to target lacZ to the sensory neurons and thereby described the anatomy of a subset of femoral chordotonal neurons and their axonal projections to the CNS. Using the same P[Gal4] insertion to target expression of the tetanus toxin light chain, we demonstrated that this set of sensory neurons was necessary for normal function of the reflex (Reddy et al., 1997; Trimarchi et al., 1998).

The present study uses the same $\mathrm{P}[\mathrm{Gal}$ ] $]$ insertion lines to target the expression of mutant CaMKII transgenes to the sensory neurons in this reflex circuit to study habituation. Expression of various mutant CaMKII genes in the sensory neuron synapses eliminated habituation. In addition, our results demonstrate that calcium-independent activity and calcium-dependent activity of CaMKII play different roles in regulating the dynamics of this reflex. Expression of the calcium-dependent form lowers the initial responsiveness of the reflex and enhances facilitation. Expression of the calcium-independent form dramatically upregulates the response, and no facilitation or depression can be detected.

\section{MATERIALS AND METHODS}

Fly stocks. All flies were raised on standard Drosophila medium. The Gal4 line, c362, was maintained as a homozygous stock and was used to express CaMKII transgenes in the feCO sensory neurons (Reddy et al., 1997). The construction of the ala gene (Griffith et al., 1993) and the T287D and T287A mutants has been described previously (Wang et al., 1998). For the production of transgenic flies, cDNAs were cloned into the pUAST vector (Brand and Perrimon, 1993) at the polycloning site. Transgenic flies were made by using the jump-start method (Robertson et al., 1988). Three lines of upstream activating sequences fused to CaMKII (UAS-CaMKII) transgenic flies were maintained as homozygous stocks: (1) UAS-T287D (10A) (on chromosome 3), which has a T287 $\rightarrow$ D287 mutation, making the kinase calcium-independent; (2) UAS-T287A (1C3D) (on chromosome 1), which has a T287 $\rightarrow$ A287 mutation, making the CaMKII incapable of becoming calcium-independent; and (3) a transgene coding for a peptide (UAS-ala on chromosome 3 ), which is an inhibitor of CaMKII. These UAS transgenic flies were generated in the w-CS genetic background. The experimental flies were the F1 flies generated by crossing homozygous male P[Gal4]-c362 flies (insert on chromosome 3) to virgin female UAS-CaMKII flies of one of the above stocks. There were three types of control fly: (1) the wild-type Canton-S flies, (2) UAS-CaMKII flies crossed to Canton-S flies, and (3) the P[Gal4]-c362 line crossed to Canton-S. The statistical analysis compared each mutant CaMKII construct with the appropriate UASCaMKII construct crossed to CS to minimize differences in genetic background. However, all three control stocks gave similar habituation responses to repetitive stimuli (Table 1).

Histology. To examine the sensory axon projection, we crossed a recombinant c362 line carrying the Gal4 insert and the lac $Z$ gene on the same chromosome to the UAS-CaMKII lines. The tissue-specific expression of $\beta$-galactosidase was revealed by using antibodies to $\beta$-galactosidase, as previously described (Reddy et al., 1997). To examine the chordotonal organ, we processed legs according to the protocol of Reddy et al. (1997). The femur was embedded in LR White (hard) methacrylate resin at room temperature and sectioned length-wise at 3 $\mu \mathrm{m}$ thickness on a rotary microtome with a steel blade. Sections were mounted on glass slides coated with $2 \%$ Formvar and counterstained with hematoxylin and eosin.

Behaviors. Headless flies perform a number of behaviors that can be used to assay the general function of the thoracic nervous system. We routinely observed the following behaviors in the headless flies: (1) righting reflex: when flies were placed on their backs, the headless flies could right themselves and maintain an upright position; (2) cleaning reflex: with tactile stimulation to the thoracic bristles, the decapitated fly cleans, with a patterned leg movement, the position covered by the stimulated bristles (Vandervorst and Ghysen, 1980).

Electrophysiology. Adult flies 2-5 d old, male or female, were anesthetized on ice for 5-10 min and decapitated. The flies were left in a moist Petri dish for at least $1 \mathrm{hr}$ to allow for recovery from surgery. A fly was mounted at the edge of a wax platform, with the tibia and tarsi of the mesothoracic leg hanging free over the edge of the platform. The femur was stabilized by waxing its distal end to the platform, and a ground electrode was inserted into the abdomen of the fly. To stimulate the femoral chordotonal organ, we moved the tibia by a metal loop that was placed around it and that was driven by a small speaker. The initial FT joint angle was $138.6 \pm 1.08^{\circ}$ (mean \pm SEM), and the movement amplitude was $25.7 \pm 1.26^{\circ}$.

To record the excitatory junction potentials (EJPs) from the tibial extensor muscle, we inserted a sharpened tungsten recording electrode or a glass electrode filled with modified Drosophila saline (Trimarchi and Murphey, 1997) into the tibial extensor muscle from the dorsal aspect of the femur. Data were recorded with a Getting amplifier (Getting Instruments, Iowa City, IA), digitized on-line, and stored on a computer with the pClamp 6.02 program (Axon Instruments, Foster City, CA).

To induce habituation, we applied 15 cycles of sine wave stimulation at $2 \mathrm{~Hz}$ to the tibia. For each preparation the 15 cycle stimulus was repeated 10 times, with an intertrial interval of $60 \mathrm{sec}$. To quantify the reflex response, we set a spike detector threshold approximately three times larger than the background level (see Fig. $1 B$, third myogram trace) and counted the number of EJPs per $50 \mathrm{msec}$. Control and treated specimens 

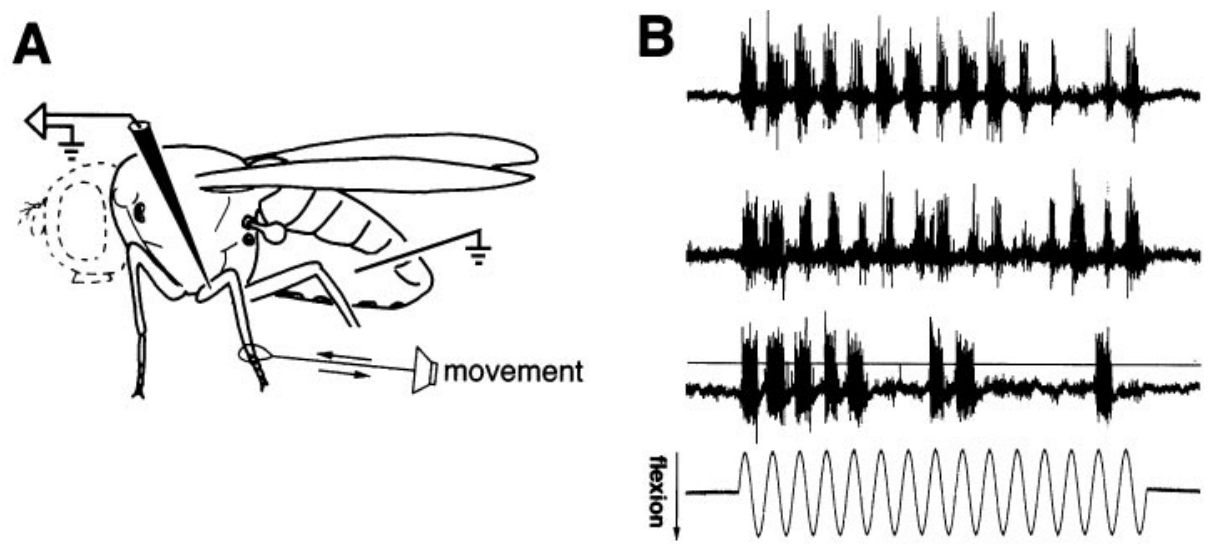

\section{C}

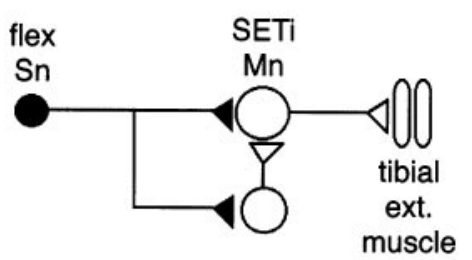

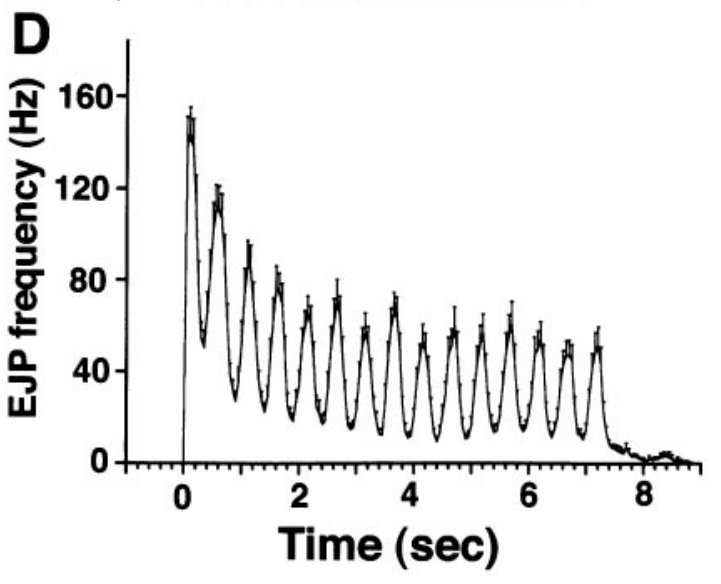

Figure 1. Habituation of the resistance reflex. $A$, Schematic of the recording situation. A decapitated fly was waxed to a small platform, with the femur of the middle leg immobilized and the tibia extended over a ledge and free to move about the tibiofemoral joint. The tibia was flexed and extended rhythmically by a movement generator at a frequency of 2 $\mathrm{Hz}$. Myograms were recorded from the tibial extensor muscle. $B$, Three representative examples of the myograms from a single preparation are shown, and the corresponding movement is shown in the bottom trace ( $2 \mathrm{~Hz}$ sine wave). A spike detector threshold was set as illustrated in the third myogram trace. $C$, Schematic of the neural circuit underlying the resistance reflex. The synapses of the flexionsensitive units in the chordotonal organ are altered by the CaMKII constructs ( filled symbols). This circuit is based on our work in Drosophila (Reddy et al., 1997) and work in other insects (Burrows, 1987; Field and Pfluger, 1989; Bassler, 1993). $D$, Graphic representation of the average frequency of EJPs per $50 \mathrm{msec}$ interval. Ten trials consisting of 15 flexion movements were obtained from each specimen, and then the frequency of EJPs was averaged over all specimens; the error bars indicate SEM from 32 wild Canton-S flies. The peak junction potential rates corresponding to each flexion were determined and fit with a single exponential function (see Figs. 3-5 and Materials and Methods). were alternated throughout this work to control for variables such as room temperature.

Data analysis and statistics. Statistical analysis of the habituation of the resistance reflex focused on variations in the peak responses over time. The values of the peaks used in the modeling of exponential decay were extracted from the averages of the replicate profiles within the control and experimental groups, respectively. We used a first-derivative approach in which a change in the slope of the profile over time from positive to negative identified a peak. Occasionally, the first peak could not be identified in this way, and we then chose the maximum of the five points. Group differences were assessed for statistical significance with ANOVA and the simple exponential decay model (see Fig. 3). In our formulation of the exponential decay function, $y=\alpha \exp [-\beta t]$, where $t$ was time, $y$ was peak EJP frequency, $\beta$ was the rate of decay, and $\alpha$ was the $y$ intercept. Nonlinear least-squares estimation was used to obtain the estimates of both $\alpha$ and $\beta$. Adequacy of the simple exponential decay model in explaining the observed variation was reported by using the $R^{2}$ statistic, defined as the percentage of variance explained. For each comparison of control versus experimental we tested three hypotheses: (1) overall equality of the two fitted curves of exponential decay, using a $2^{\circ}$ of freedom Wald $\chi^{2}$ statistic; (2) equality of the mean of the first extracted peak, using ANOVA; and (3) equality of mean of the plateau of the last five peaks, using ANOVA. All analyses were performed with the SAS statistical software for personal computers (SAS Institute, 1989).

\section{RESULTS}

\section{Habituation of the resistance reflex}

Flexing the femorotibial joint elicited a resistance response from the tibial extensor motor neurons, and this reflex response decayed on repetition (Fig. 1). The frequency of EJPs was monitored throughout a repetitive series of sinusoidal flexion-extension movements, and the data were collected in $50 \mathrm{msec}$ bins (the average and SEM are shown as the vertical bars in Fig. 1D). The data for 32 wild-type specimens demonstrated that the peak response corresponded to the flexion phase of the movement.
With $2 \mathrm{~Hz}$ of stimulation the peak response decayed smoothly, with a time constant of $1 \mathrm{sec}$ in these examples (the range for various groups of control specimens was 1-2 sec) to a plateau of $\sim 40 \%$ of the initial response (Figs. $1 D, 3 B$, filled symbols). This response was readily fit with a single exponential decay function, as is the case for many other examples of habituation.

This response decrement conformed to other features of habituation as well (Jin et al., 1998). When the $2 \mathrm{~Hz}$ stimulus was terminated, the initial response recovered spontaneously to $\sim 75 \%$ of the initial amplitude in $2 \mathrm{sec}$ and was fully recovered within $60 \mathrm{sec}$. As is the case for most other examples of habituation, the rate of decrement in this reflex was dependent on the frequency and amplitude of the stimulus. For example, decreasing the rate of flexion from 2 to $0.5 \mathrm{~Hz}$ increased the time constant of decay from 1 to $22 \mathrm{sec}$. The time constant also was correlated with stimulus strength. In one group of experimental animals a $25^{\circ}$ flexion led to a time constant for decay of $1.9 \mathrm{sec}$. Reducing the stimulus strength by reducing the flexion movement to $20^{\circ}$ produced a time constant of $1.3 \mathrm{sec}$. Finally, the response could be dishabituated by tactile stimuli to the abdomen. When an animal was stimulated with 15 successive flexion movements and a tactile stimulus was provided during the ninth and tenth stimulus, the response recovered to $\sim 60 \%$ of the initial response; this recovery was statistically significant.

The myograms were obtained from the tibial extensor muscle, which appeared to be innervated by two motor neurons: the well characterized fast extensor of the tibia (FETi) (Trimarchi and Schneiderman, 1993; Trimarchi et al., 1998) and the smaller slow extensor of the tibia (SETi). The FETi seldom reached threshold in our experiments, and most, if not all, of the data could be 


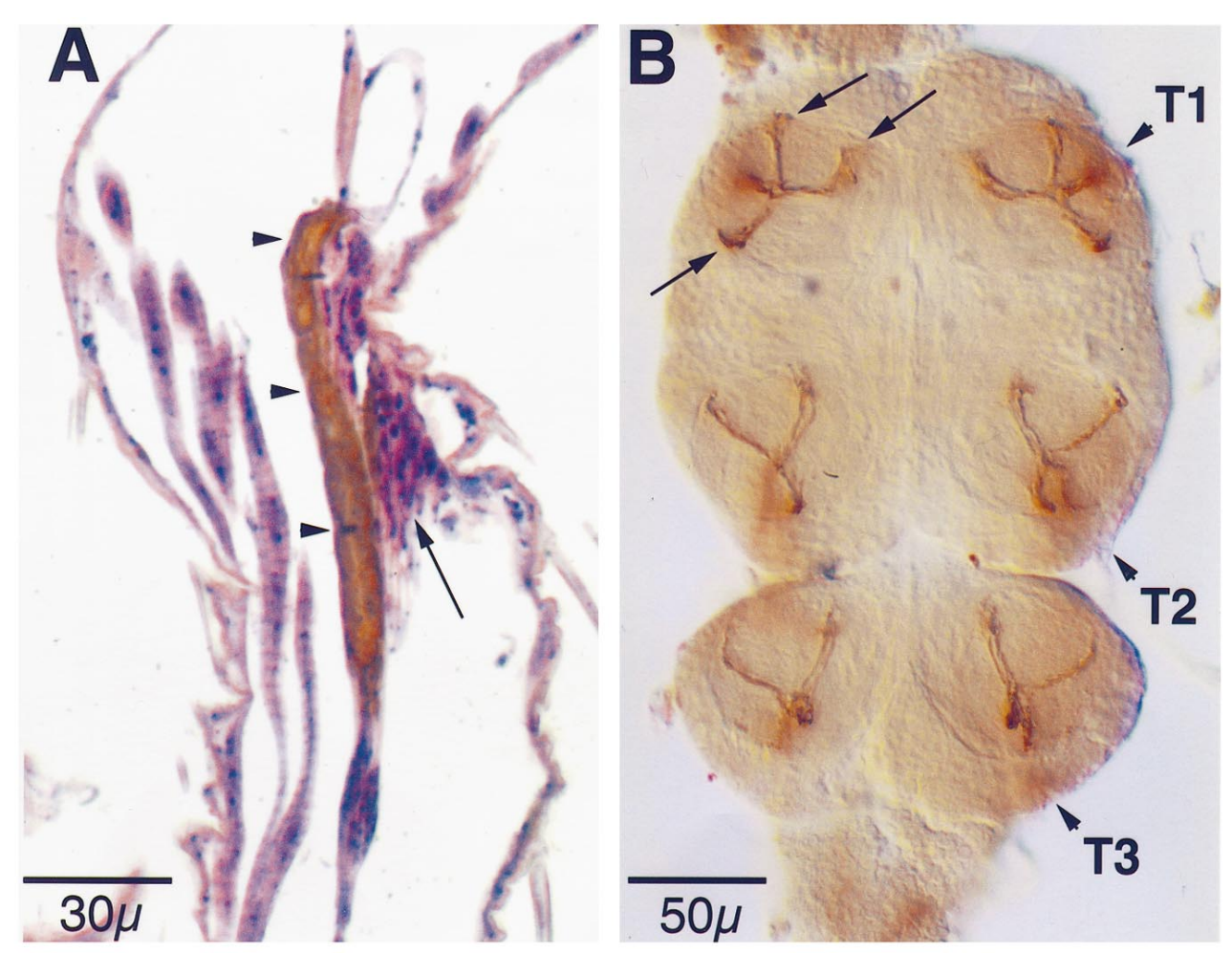

Figure 2. The anatomy of the sensory neurons that drive the resistance reflex. $A$, The anatomy of the femoral chordotonal organ. This section, parallel to the long axis of the leg, demonstrates that the $\mathrm{P}[\mathrm{Gal} 4]-\mathrm{c} 362$ insert is expressed only in a subset of the feCO somata. The brown label is the DAB reaction product in the expressing cells (arrowheads), and the purple staining of somata immediately to the right is the unlabeled portion of the feCO (arrow). A few muscle fibers of the tibial extensor muscle are seen to the left of the feCO. $B$, The axonal arborizations of the feCO axons in the thoracic nervous system of a wild-type fly. The axons enter the CNS from each leg nerve (arrowheads), divide, and terminate in three collaterals in each neuromere (filled arrows). The recordings of Figure 1 were obtained from the leg controlled by the axons in the second thoracic segment (T2). $C$, Wild-type feCO sensory axon projection in the first thoracic neuromere. The feCO sensory axons from the first leg project into the corresponding first thoracic neuromere and make a characteristic threebranched projection pattern (anterior, posterior, and lateral branches; arrows). Expression of CaMKII does not disrupt sensory axon projections in the transgenic flies. $D$, UAS-ala. $E$, UAST287A. $F$, UAS-T287D.
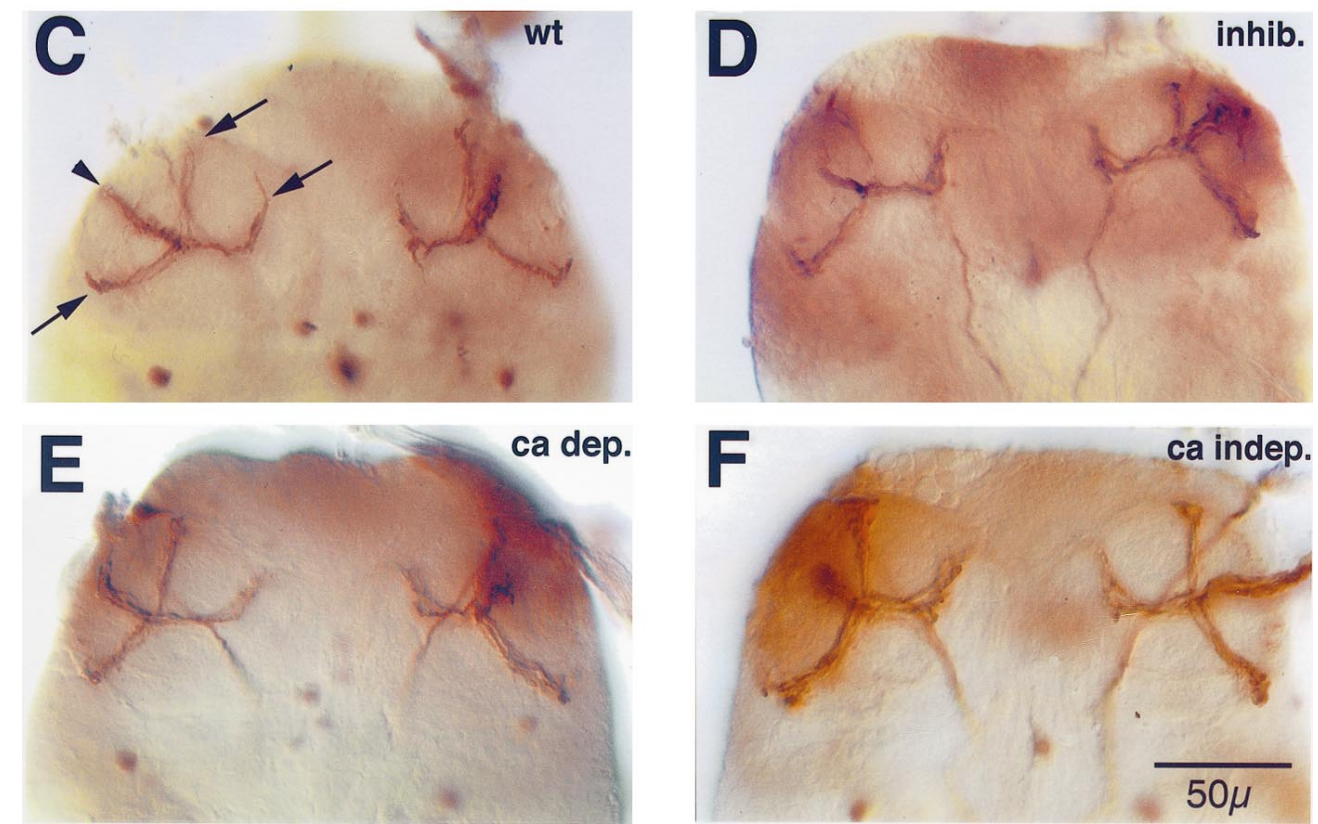

attributed to SETi (Jin, 1998; Trimarchi et al., 1998). This was consistent with data on this reflex in other insects in which only the smaller postural motor neuron was actively involved in this reflex (Field and Pfluger, 1989; Bassler, 1993).

We visualized the sensory neurons that drive this reflex, using a $\mathrm{P}[\mathrm{Gal} 4]$ insertion (P[Gal4]-c362) that was expressed in a subset of the neurons in the femoral chordotonal organ. When this $\mathrm{P}[\mathrm{Gal} 4]$ insertion was used to drive a UAS-lac $Z$ construct and the lac $Z$ was revealed with an antibody, a subgroup of the cell bodies in the femoral chordotonal was labeled (Fig. $2 A$ ). We estimated that fewer than one-half of the 140 cell bodies (Shanbhag et al.,
1992) of the feCO were labeled and that the labeled group of somata is homologous to the distal component of the feCO in other insects (Field and Pfluger, 1989; Reddy et al., 1997). The somata from each leg projected axons from the leg to the corresponding leg neuropil of the CNS, where they terminate in three collaterals (Fig. 2B, filled arrows). Targeted expression of the tetanus toxin light chain (Sweeney et al., 1995) to these sensory neurons demonstrated that these axons were necessary for normal reflex function (Reddy et al., 1997; Trimarchi et al., 1998).

The enhancer trap ( $\mathrm{P}[\mathrm{Gal} 4]-\mathrm{c} 362)$ shown in Figure 2 labeled very few central cell bodies. In some specimens a few cell bodies 
in the thoracic neuromeres are labeled (Fig. $2 C-F$ ). Based on their location and size, none of the cell bodies appears to be the extensor motor neurons. In addition, driving tetanus toxin light chain with the c362 line has no affect on the spontaneous activity recorded from the muscle. Our conclusion is that Gal4 expression, and thereby the CamKII transgene expression, is confined to the presynaptic sensory neurons underlying the reflex.

\section{The role of presynaptic CaMKII in habituation}

\section{Targeted expression of a constitutively active CaMKII}

Replacement of the threonine at position 287 with an aspartate (T287D) in the Drosophila CaMKII made the kinase calciumindependent and increased the basal level of the active kinase (Wang et al., 1998). Targeted expression of this mutant CamKII (T287D) to the feCO sensory neurons led to a strong response that was maintained, without decrement, throughout the stimulus presentation. As shown in the representative examples from a single preparation in Figure $3 A$, a strong burst of junction potentials was elicited by nearly every stimulus, and the phase of the response was correlated with flexion just as is seen in control specimens. The quantified data from seven specimens showed no apparent response decrement and demonstrated a statistically significant difference between the experimental flies and the control flies (Fig. 3B; Table 1). For a quantitative comparison between mutant and control flies we used flies resulting from a cross between Canton-S and the line carrying the appropriate UAS-CaMKII construct as a control (Figs. $3 B$, filled symbols). As an added control we tested flies resulting from a cross of the $\mathrm{P}[\mathrm{Gal} 4]-\mathrm{c} 362$ line and CS flies and showed that they too habituated normally with a decay constant of $\sim 1.9 \mathrm{sec}$. The results suggest that habituation was eliminated by increasing the calciumindependent CaMKII activity in the presynaptic sensory neurons.

There was a ceiling effect in these data, because a maximum motor neuron firing rate of $\sim 120 \mathrm{~Hz}$ was observed. We could see no significant difference between the first burst for the experimental and control flies. However, in the experimental flies the motor neuron firing rate remained at this apparent ceiling throughout the stimulus. To assess this ceiling effect further, we used two different stimulus strengths in another group of seven T287D animals (Fig. 3C). Each animal was tested with a series of $26^{\circ}$ flexion movements, and then the stimulus strength was decreased to $20^{\circ}$. With the weaker stimulus the overall response shifted downward and was significantly different from the strong stimulus [the slope was significant for the weak stimulus ( $p=$ $0.09)$, but not for the strong stimulus $(p=0.32)]$. These results with the T287D construct suggested that CaMKII adjusts the basal level of transmitter release, and this adjustment is affecting the dynamics of the sensory synapses (see Discussion).

\section{Expression of CaMKII is incapable of becoming calcium-independent}

We tested the role of CaMKII autophosphorylation in habituation by targeting the expression of a mutant CaMKII transgene that was incapable of autophosphorylation at position 287 (a threonine-to-alanine substitution, T287A) to the sensory neurons. The reflex was modulated in three ways when this construct was expressed in sensory neurons. As shown by the representative examples, the first response was reduced dramatically (Fig. $4 A 1, B$, asterisks). We determined the average peak firing rates during the first movement for each animal and then compared the treated and the control groups; they were significantly different (Table 1). This low initial response was followed by a dramatic sensitization of the reflex response after the initial burst (Fig. $4 B$, open symbols). Analysis of the data for the control flies showed that the decrement could be described as an exponential decay (Fig. 4B, filled symbols), and the data for the experimental specimens were significantly different from this $(p<0.0001)$. When we analyzed the plateau levels in the T287A transgenic flies, they were significantly greater than the plateau levels for wild-type flies $(p<0.01)$. Our interpretation of these results is that expression of the calcium-dependent form of CaMKII decreased the basal activity level of CaMKII, and this decrease altered the initial response. However, the addition of CaMKII by targeted expression increased the overall response plateau and decreased the response decrement (see Discussion).

\section{Inhibiting CaMKII in the sensory neurons}

To assess the effects of CaMKII on habituation further, we targeted the expression of a peptide inhibitor of CaMKII (ala) to the sensory neurons. Habituation effectively was eliminated in these animals, although the overall response plateau was higher than with controls (Fig. 5, Table 1). The sample myograms also showed that targeted expression of ala significantly reduced the initial response to a flexion stimulus (Fig. 5A, asterisk; $p<$ $0.0001)$. The low initial response was followed by an immediate sensitization between the first and second stimuli. This early sensitization of the response also was seen in other contexts. Animals carrying the T287D construct exhibited a similar sensitization when they were stimulated weakly (see Fig. $3 C$ ), as did wild-type animals (Jin, 1998).

\section{Normal axon projection of the feCO sensory neurons in the CaMKII mutant flies}

We considered the possibility that the altered habituation of the resistance reflex was a secondary consequence of altered axonal growth caused by interfering with the CaMKII signal transduction pathway during metamorphosis. To assess the anatomical structure of the sensory neurons, we drove a UAS-lacZ construct in parallel with the CaMKII constructs. Each of the mutant constructs was tested, and we observed no anatomical change of the sensory neuron axon projection; the characteristic tripartite branching pattern of the wild-type sensory neurons was present in all three strains of the CaMKII transgenic flies (see Fig. $2 C-F$, filled arrows). Our interpretation of the light microscopic data was that no change in structure was induced by these various CaMKII constructs. Because the P[Gal4]-c362 construct was activated after the axons had grown into the CNS (R. Murphey, unpublished data), we wondered whether earlier activation would have a different effect. Using a Gal4 insert that was expressed during the growth of these axons, we drove the various CaMKII constructs earlier in the development of the reflex circuit, and this also failed to alter the sensory axon projection (data not shown). In summary, we could find no change in the anatomy of the afferent projections caused by the various manipulations, and we concluded that the physiological results illustrated in Figures 3-5 were attributable to changes in synaptic function per se.

\section{Normal cleaning reflex in the CaMKII transgenic flies}

As a control for the overall health of the flies and to assess other aspects of thoracic ganglion function in these flies, we examined an easily assayed cleaning reflex. It has been shown that headless flies are able to maintain a normal posture and perform reflexes, including a cleaning reflex (Vandervorst and Ghysen, 1980; Corfas and Dudai, 1989). We examined the CaMKII transgenic flies 


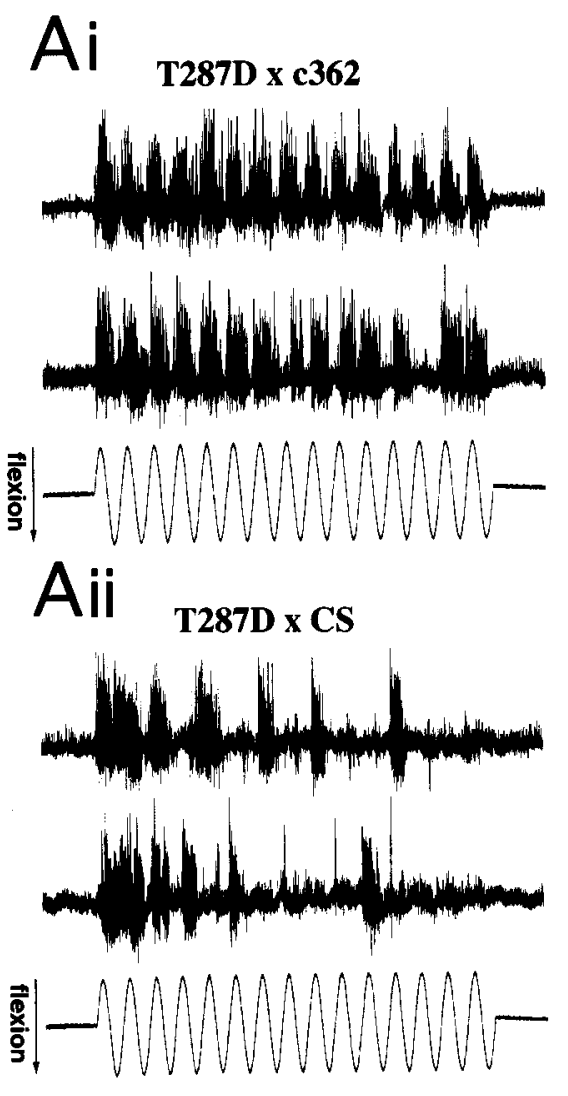

B

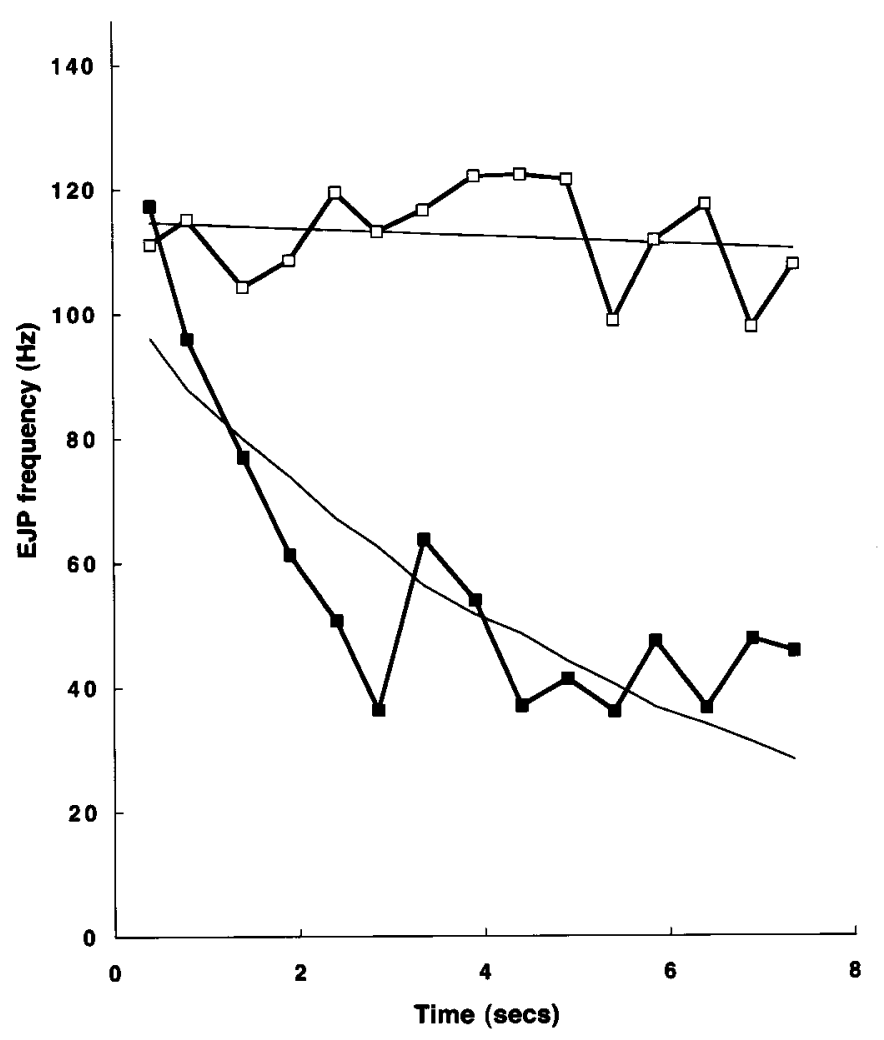

C

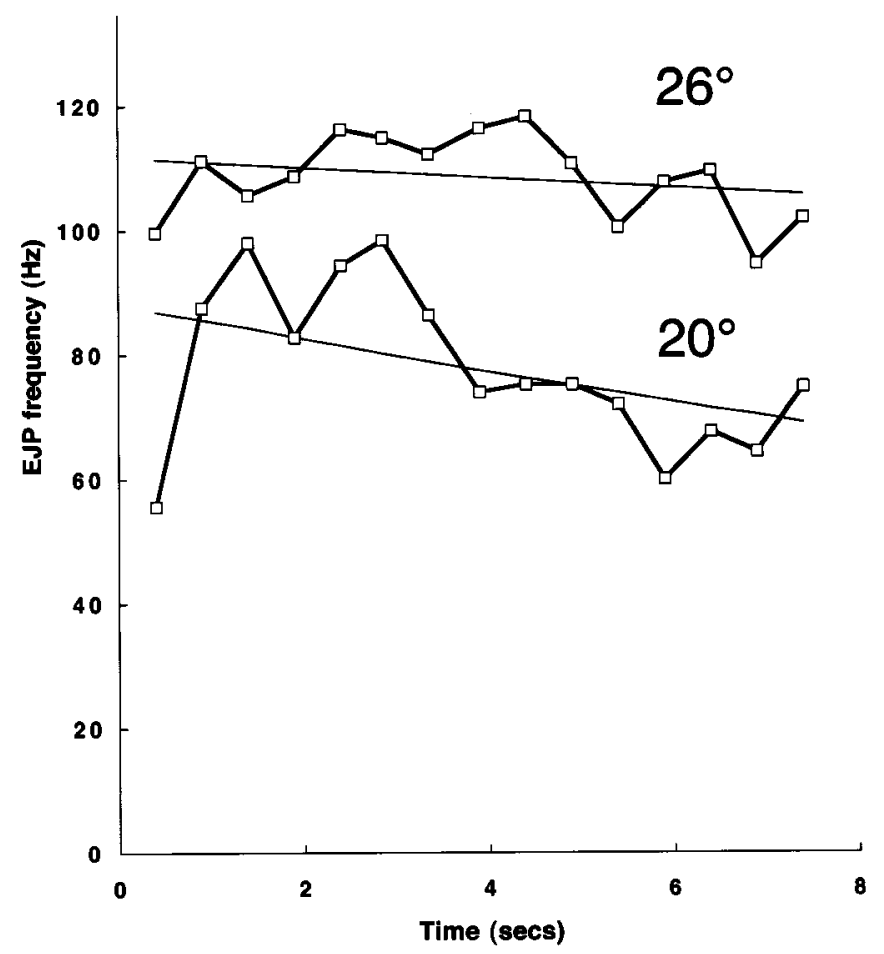

Figure 3. Targeted expression of the constitutively active CaMKII (T287D) in the presynaptic sensory neurons increased the reflex response and blocked habituation. $A i$, Representative recordings of the resistance reflex in flies expressing the T287D transgene. Note the continuous bursting pattern seen throughout the series of rhythmic flexion movements. Aii, Sample myograms from the genetic control flies $(T 287 D \times C S)$. Note that the later bursts tend to be weaker. $B$, The average peak response to each movement showed the complete blockade of habituation in the T287D flies (open symbols). The control specimens, obtained from a cross of the UAS-T287D line with Canton-S, showed the normal habituation ( filled symbols). (Figure legend continues) 
$\mathrm{Ai}$
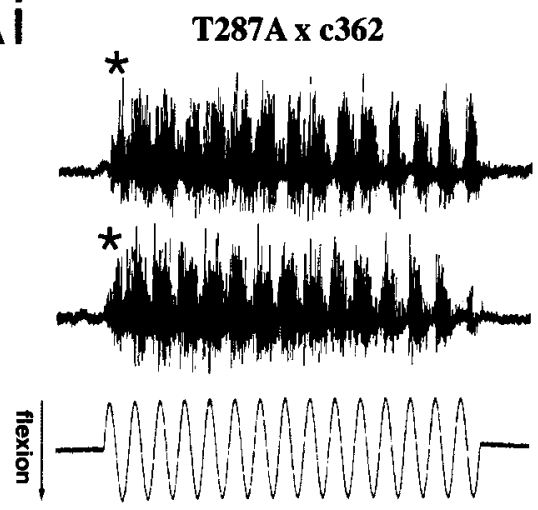

Aii

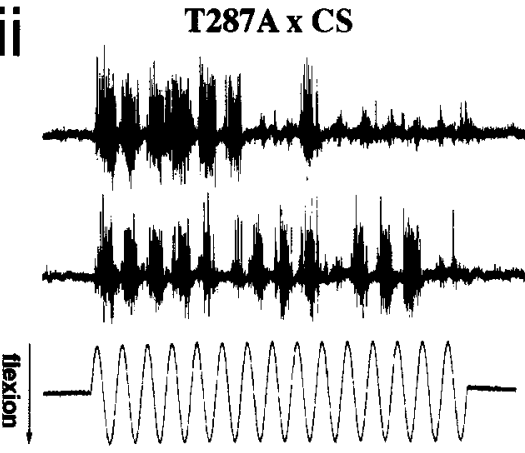

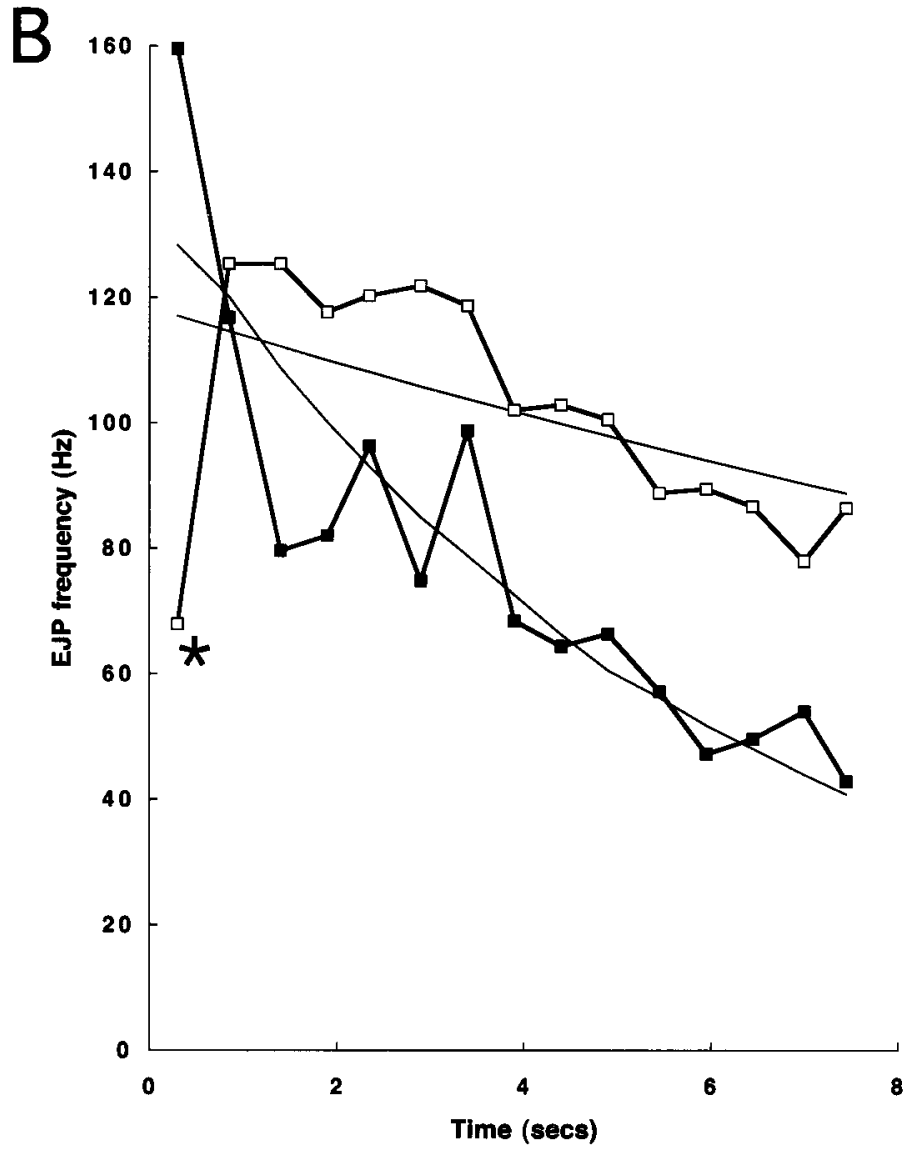

Figure 4. Targeted expression of a CaMKII construct incapable of autophosphorylation (T287A) in the presynaptic sensory neurons. $A i$, Sample myograms from a specimen expressing the T287A transgene in the sensory neurons resulted in a reduction of the first response (asterisk), followed by a dramatic facilitation; the habituation was nearly absent. Aii, The genetic control flies showed a normal first response and normal habituation. B, The averaged data from seven T287A specimens (open symbols) showed a significant decrease of the first response (asterisk) as well as a much stronger than normal response on the remaining trials. The control specimens ( filled symbols) showed the usual response decrement (see Table 1).

for the cleaning reflex to test the idea that the targeted expression was relatively specific and did not affect the cleaning response. The bristles on the tegula of the fly were stimulated 10 times every $5 \mathrm{sec}$. In the CaMKII transgenic flies $>95 \%$ of the stimuli were effective in triggering the cleaning reflex, and these responses were no different from wild-type flies. This suggests that the function of the thoracic nervous system was primarily normal. An additional control was to assay the righting reflex in these transgenic flies. If headless wild-type flies are inverted, they immediately right themselves. The transgenic flies behaved similarly, righting themselves quickly when inverted. We conclude that the altered habituation of the resistance reflex was specific to the targeted sensory neurons and was not attributable to a more general change in nervous system function.

\section{DISCUSSION}

The results showed three main points. First, alteration of sensory neuron function in Drosophila by targeted expression of CAMKII transgenes disrupted habituation of a simple reflex.
This suggested a presynaptic locus for habituation. Second, expression in the presynaptic sensory neurons highlighted an important role for presynaptic CaMKII in controlling short-term synaptic plasticity. Third, mutant forms of the kinase distinguished a role for the calcium-dependent and calciumindependent forms of the kinase. It appeared that the basal level of the calcium-independent form set the overall transmitter release levels and thereby determined the rate of habituation.

\section{A presynaptic role for CaMKII}

Previous studies of CaMKII and learning could not distinguish between pre- and postsynaptic effects because of the nature of the genetic manipulation. In mice, a complete knock-out of the gene meant no cells expressed the kinase (Silva et al., 1992a,b); in flies, heat-shock induction of a CaMKII inhibitor targeted the inhibitor to all tissues (Griffith et al., 1993). Recently, the use of more sophisticated targeting systems has allowed a clear demonstration of the role of CaMKII in the postsynaptic cells. Spatially controlled expression of a constitutively active CaMKII in the

The control and treated curves are significantly different $(p<0.0001) . C$, In a separate group of specimens the strength of the stimulus was adjusted by changing the angle of the movement in T287D specimens. The stronger stimulus $\left(26^{\circ}\right.$ movements) showed no habituation, the weaker stimulus $\left(20^{\circ}\right)$ revealed an underlying habituation of the response, and the curves were significantly different $(p<0.0001)$. Note the similarity in the shape of the response between the weak response seen here and the shapes of the curves for T287A (see Fig. 4) and the ala specimens (see Fig. 5, Table 1). 

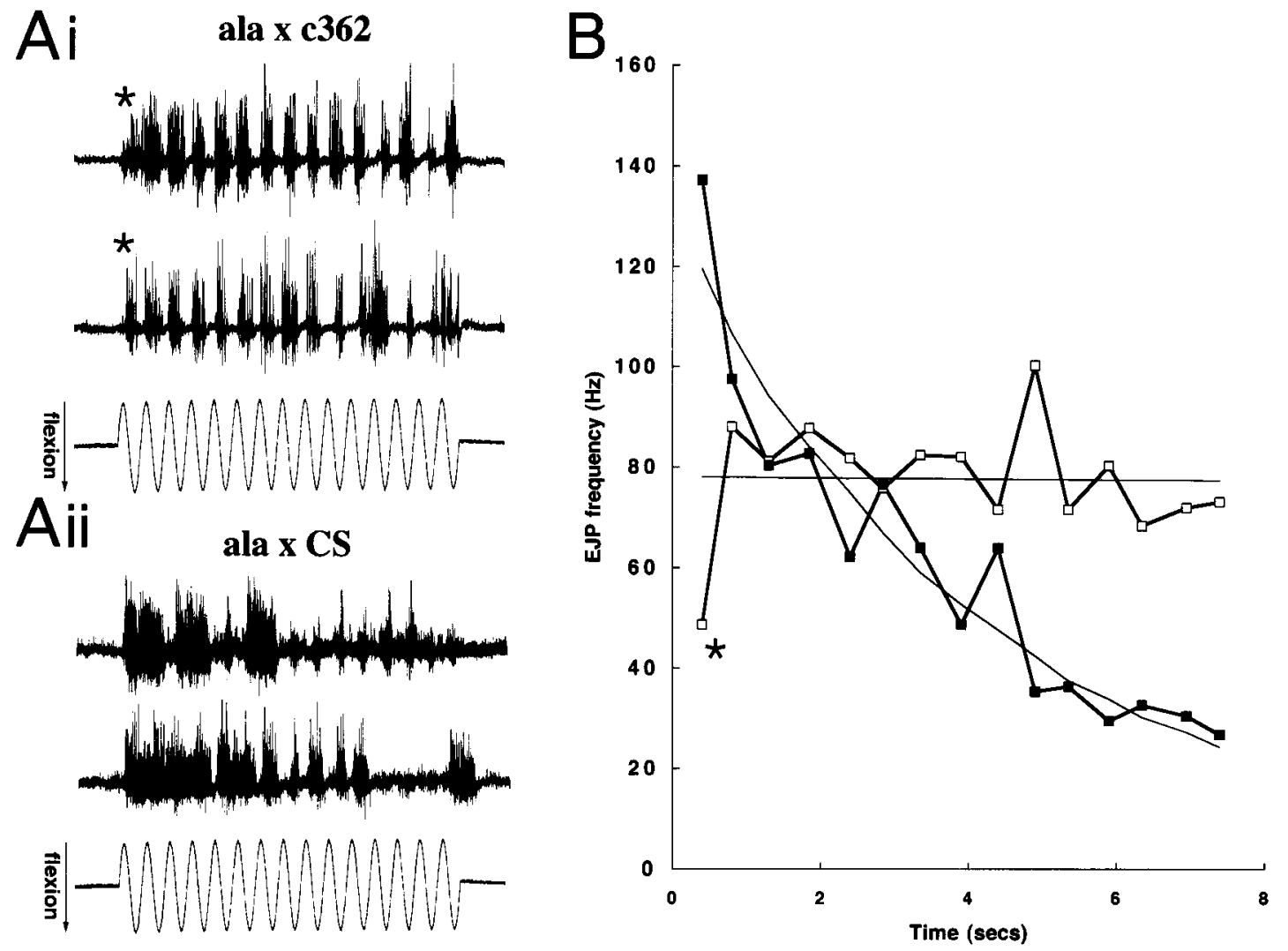

Figure 5. Targeted expression of a CaMKII inhibitory peptide (ala) decreased reflex response and blocked habituation. Shown are examples of the myograms in treated $(A i)$ and control specimens (Aii). Note the decreased first burst in the experimental animals (asterisks). B, The averaged peak response from seven experimental preparations (open symbols) showed reduced reflex response and lack of habituation as compared with controls ( filled symbols). The initial response was only $33 \%$ of the response in the control flies. Note that the plateau level of peak junction potential rate was $\sim 80 \mathrm{~Hz}$ significantly above the wild-type data $(p<0.0001)$. The wild-type data are shown as filled symbols (see Table 1).

postsynaptic pyramidal neurons in the hippocampus altered the frequency response at the CA3-CA1 synapses. The synapses became more prone to long-term depression at intermediate frequencies (Mayford et al., 1995, 1996). This postsynaptic change was associated with an impaired ability in a spatial learning task (Bach et al., 1995).

The presynaptic function of CaMKII has been studied in relatively few preparations, and none of these studies has examined the dynamics of transmitter release. In the squid, injection of pharmacological agents disrupted CaMKII function and altered neurotransmitter release (Llinás et al., 1985). Subsequent studies led to the proposal that phosphorylation of synapsins by CaMKII frees synaptic vesicles from a reserve pool and thus increases vesicular release (Greengard et al., 1993). Finally, ongoing studies at the Drosophila larval neuromuscular junction involving expression of a mutant CaMKII presynaptically demonstrate a role for CaMKII in transmitter release at this synapse (M. Coleman and L. Griffith, unpublished data).

In the present study the genetic manipulation of CaMKII was restricted to the presynaptic sensory neurons, and habituation was severely disrupted. We assumed that, as in many other preparations (Castelluci et al., 1970; Zucker, 1972), habituation was the result of homosynaptic depression of transmitter release at the sensory synapses, and our analysis of habituation was consistent with this idea (Jin, 1998). The results for targeted expression of CaMKII supported this idea because expressing various CaMKII constructs only in the sensory neurons changed habituation rates.
An accurate interpretation of our results depended on the selectivity of the targeting system. The Gal4 line we used to target transgenes was expressed primarily in sensory neurons of the femoral chordotonal organ (see Fig. 2). Targeted expression of tetanus toxin light chain in these sensory neurons blocked the resistance reflex and thereby demonstrated the crucial role of these neurons in the reflex (Reddy et al., 1997). Reflexes that depended on other sensory neurons such as the scratch reflex were unaffected, attesting to the selectivity of the targeting system (Trimarchi et al., 1998). In addition, the temporal profile of expression of the targeting element showed that it was activated after the sensory neurons had established their characteristic branches within the CNS (R. Murphey, unpublished data). Consistent with this late expression, our anatomical analysis at the light microscope level indicated that the anatomical connectivity diagram was normal, although we could not eliminate the possibility of ultrastructural changes in the presynaptic sensory axon terminals.

The targeting system did not affect the postsynaptic cells and did not appear to be expressed there. There was no indication of lacZ expression in motor neurons (see Fig. 2), and expression of the tetanus toxin light chain by the same Gal4 enhancer trap did not block spontaneous myogram activity in the extensor muscle, indicating that the motor neurons were functioning normally, although the sensory neurons were blocked (Reddy et al., 1997). In summary, the selectivity of the P[Gal4] insertion allowed us to express the CaMKII transgenes in the relevant sensory neurons, 
leaving CaMKII undisturbed in the other sensory neurons and the motor neurons.

\section{The role of calcium-independent and calcium- dependent CaMKII in reflex response and reflex habituation}

Autophosphorylation renders CaMKII calcium-independent, thereby prolonging its activation well beyond the duration of the original calcium signal (Hanson and Schulman, 1992). A threonine-to-aspartate mutation at position 287 (286 in mammals) mimics autophosphorylation at that position. The T287D mutant CaMKII expresses 30-80\% of wild-type maximal activity in the absence of calcium (Braun and Schulman, 1995). This property led to the proposal that CaMKII can serve as a molecular memory device (Miller and Kennedy, 1985; Lisman and Goldring, 1988). Subsequent experimental evidence indicated a correlation between long-lasting synaptic modification and prolonged calcium-independent CaMKII activity. In the hippocampus, LTP-inducing stimuli were shown to increase calciumindependent CaMKII activity and T286 autophosphorylation (Fukunaga et al., 1993; Barria et al., 1997; Ouyang et al., 1997). Direct evidence for a role for autophosphorylation at T286 in the synaptic plasticity associated with learning was provided recently by showing that a point mutation at Thr286 blocked LTP in the hippocampus and affected learning (Giese et al., 1998).

We sought to determine the mechanism for CaMKII activity on synaptic dynamics by comparing the targeted expression of the calcium-dependent and calcium-independent transgenes in the presynaptic terminal. Increasing the total CaMKII activity with either a calcium-independent kinase (T287D) or calciumdependent kinase (T287A) reduced or eliminated habituation. The manner in which each of these manipulations eliminated habituation differed and may indicate one aspect of CaMKII action in these presynaptic neurons. Increasing the amount of calcium-independent kinase activity with the T287D construct eliminated habituation, and the response level remained at its maximum throughout the stimulus trial (see Fig. $3 B$ ). This implies that the additional kinase activity was able to mobilize transmitter release and relieve the presynaptic depression that was normally responsible for habituation. Even with reduced stimulus strength that enhances depression in most habituation paradigms, we saw only a weak depression. An increased amount of calcium-dependent activity with the T287A construct had a similar effect, slowing the rate of depression and supporting the idea that additional CaMKII activity can relieve habituation.

There was one important difference between the calciumdependent and the calcium-independent constructs; the initial response was much weaker than normal for the calciumdependent construct (T287A; see Fig. 4, asterisks). This weak first burst was followed by a rapid sensitization phase that brought the response up to the very high levels seen with the calciumindependent form. The initial weak response seen with expression of the calcium-dependent kinase (T287A) may be attributable to an induced change in the basal level of calcium-independent activity in the presynaptic neuron. Calcium-independent activity of CaMKII is generated by autophosphorylation of T287. This phosphorylation of T287 is an intersubunit reaction within the CaMKII holoenzyme, meaning that the activity of the neighbor of a particular subunit is of critical importance in its phosphorylation (Hanson et al., 1994; Wang et al., 1998). The incorporation of the T287A subunit into the CaMKII holoenzyme is likely to reduce this basal level of calcium-independent kinase activity
(Molloy and Kennedy, 1991). In flies expressing T287A the autophosphorylation of wild-type subunit could be compromised by the presence of the mutant subunit in the holoenzyme. In this way the basal level of calcium-independent activity would decrease even if the level of endogenous wild-type kinase was unchanged, and this would decrease the initial response. This idea is consistent with the lowered initial response that is seen when the inhibitory peptide ala is expressed presynaptically. Because the ala construct directly inhibits the catalytic activity of CaMKII, we suggest that the lowered initial response is attributable to a decrease in basal CaMKII activity (see Fig. 5).

We considered the possibility that overexpression of CaMKII could lead to these effects. However, overexpression per se cannot be used to explain the dramatic difference between 287A and 287D. Both are presumed to be "overexpressed" on the wild-type background, but they give very different responses at the beginning of stimulation, as described above. The 287A construct decreased the initial response, but the 287D construct did not. This difference between targeted expression of the two different constructs strongly suggests that the constructs are working in distinct ways, and the results cannot be explained in a manner simply attributable to overexpression. A wild-type transgene is being constructed and could be used to confirm this idea.

Taken together, the data are consistent with a model in which the basal level of calcium-independent CaMKII in the presynaptic neuron sets the response level of the synapse and controls the expression of appropriate response dynamics according to the activity history of the synapse. Existing biochemical data suggest that the basal level of calcium-independent CaMKII activity is crucial to basal synaptic function. If the basal level of kinase is correlated with the size of the immediately releasable pool, then transmitter release might be adjusted upward as a function of active kinase concentration. When the basal level is lowered by T287A, the size of the immediately releasable pool will decrease and the first burst will be lowered. Similarly, in the presence of the inhibitor (ala), the initial response will be suppressed. The lowrelease synapses created by expressing T287A or ala now might exhibit facilitation such as is seen at many low-release synapses (Hill and Jin, 1998). However, the sustained response will still be enhanced as compared with that seen in controls because of the increase in calcium-dependent kinase activity.

Previous studies of response decrement at identified synapses in other insects are consistent with this model. At identified synapses in the cricket giant fiber system, response decrement occurs more readily at synapses where spontaneous activity is low. In contrast, spontaneously active sensory synapses, and the interneurons they drive, do not habituate as readily (Chiba et al., 1992; Hill and Jin, 1998). In addition, experimentally increasing the sensory afferent activity during development reduced habituation of the interneuron (Murphey and Matsumoto, 1976). Our results suggest a model whereby recent activity of the sensory neurons adjusts the basal levels of the autophosphorylated form of CaMKII, and this in turn adjusts transmitter release levels and the gain and the dynamics of these synapses. Such a mechanism would provide the circuit with a "memory" of recent activity levels and set the gain of the circuit.

\section{REFERENCES}

Asztalos Z, Wegerer J, Wustmann G, Dombradi V, Gausz J, Spatz H-C, Friedrich P (1993) Protein phosphatase 1-deficient mutant Drosophila is affected in habituation and associative learning. J Neurosci 13:924-930.

Bach ME, Hawkins RD, Osman M, Kandel ER, Mayford M (1995) 
Impairment of spatial but not contextual memory in CaMKII mutant mice with a selective loss of hippocampal LTP in the range of the frequency. Cell 81:905-915.

Barria A, Muller D, Derkach V, Griffith LC, Soderling TR (1997) Regulatory phosphorylation of AMPA-type glutamate receptors by CaMKII during long-term potentiation. Science 276:2042-2044.

Bassler U (1993) The femur-tibia control system of stick insects-a model system for the study of the neural basis of joint control. Brain Res Rev 18:207-226.

Brand AH, Perrimon N (1993) Targeted gene expression as a means of altering cell fates and generating dominant phenotypes. Development 118:401-415.

Braun AP, Schulman H (1995) The multifunctional calcium/ calmodulin-dependent protein kinase, from form to function. Annu Rev Physiol 57:417-445.

Burrows M (1987) Parallel processing of proprioceptive signals by spiking local interneurons and motoneurons in the locust. J Neurosci 7:1064-1080.

Castelluci VF, Pinsker H, Kupfermann I, Kandel ER (1970) Neuronal mechanisms of habituation and dishabituation of the gill-withdrawal reflex in Aplysia. Science 167:1745-1748.

Chiba A, Kamper G, Murphey RK (1992) Response properties of interneurons of the cricket cercal sensory system are conserved in spite of changes in peripheral receptors during maturation. J Exp Biol 164: 205-226.

Corfas G, Dudai Y (1989) Habituation and dishabituation of a cleaning reflex in normal and mutant Drosophila. J Neurosci 9:56-62.

Duerr JS, Quinn WG (1982) Three Drosophila mutations that block associative learning also affect habituation and sensitization. Proc Natl Acad Sci USA 79:3646-3650.

Engel JE, Wu C-F (1996) Altered habituation of an identified escape circuit in Drosophila memory mutants. J Neurosci 16:3486-3499.

Field LH, Pfluger HJ (1989) The femoral chordotonal organ: a bifunctional orthopteran (Locusta migratoria) sense organ? Comp Biochem Physiol [A] 93:729-743.

Fukunaga K, Stoppini L, Miyamoto E, Muller D (1993) Long-term potentiation is associated with increased activity of $\mathrm{Ca}^{2+} /$ calmodulindependent protein kinase II. J Biol Chem 268:7863-7867.

Giese KP, Fedorov NB, Filipkowski RK, Silva AJ (1998) Autophosphorylation at Thr286 of the $\alpha$-calcium/calmodulin kinase II in LTP and learning. Science 279:870-873.

Greengard P, Valtorta F, Czernik AJ, Benfenati F (1993) Synaptic vesicle phosphoproteins and regulation of synaptic function. Science 259:780-785.

Griffith LC, Verselis LM, Aitken KM, Kyriacou CP, Danho W, Greenspan RJ (1993) Inhibition of calcium/calmodulin-dependent protein kinase in Drosophila disrupts behavioral plasticity. Neuron 10:501-509.

Hanson PI, Schulman H (1992) Neuronal $\mathrm{Ca}^{2+} /$ calmodulin-dependent protein kinases. Annu Rev Biochem 61:559-601.

Hanson PI, Kapiloff MS, Lou LL, Rosenfeld MG, Schulman H (1989) Expression of a multifunctional $\mathrm{Ca} / \mathrm{calmodulin}$-dependent protein kinase and mutational analysis of its autoregulation. Neuron 3:59-70.

Hanson PI, Meyer T, Stryer L, Schulman H (1994) Dual role of calmodulin in autophosphorylation of multifunctional CaM kinase may underlie decoding of calcium signals. Neuron 12:943-956.

Hill AAV, Jin P (1998) Regulation of synaptic depression rates in the cricket cercal sensory system. J Neurophysiol 79:1277-1285.

Jin P (1998) Neural plasticity and its molecular mechanisms in Drosophila. PhD thesis, University of Massachusetts.

Joiner MIA, Griffith LC (1997) CaM kinase II and visual input modulate memory formation in the neuronal circuit controlling courtship conditioning. J Neurosci 17:9384-9391.

Lisman JE, Goldring MA (1988) Feasibility of long-term storage of graded information by the $\mathrm{Ca}^{2+} /$ calmodulin-dependent protein kinase molecules of the postsynaptic density. Proc Natl Acad Sci USA 85: $5320-5324$.

Llinás R, McGuinness TL, Leonard CS, Sugimori M, Greengard P (1985) Intraterminal injection of synapsin I or calcium/calmodulindependent protein kinase II alters neurotransmitter release at the squid giant synapse. Proc Natl Acad Sci USA 82:3035-3039.
Malinow R, Schulman H, Tsien RW (1989) Inhibition of postsynaptic PKC or CaMKII blocks induction but not expression of LTP. Science 245:862-866.

Mayford M, Wang J, Kandel ER, O’Dell TJ (1995) CaMKII regulates the frequency-response function of hippocampal synapses for the production of both LTD and LTP. Cell 81:891-904.

Mayford M, Bach ME, Huang YY, Wang L, Hawkins RD, Kandel ER (1996) Control of memory formation through regulated expression of a CaMKII transgene. Science 274:1678-1683.

Miller SG, Kennedy MB (1986) Regulation of brain type II calcium/ calmodulin-dependent protein kinase by autophosphorylation, a calcium-triggered molecular switch. Cell 44:861-870.

Molloy SS, Kennedy MB (1991) Autophosphorylation of type II calcium/calmodulin-dependent protein kinase in cultures of postnatal rat hippocampal slices. Proc Natl Acad Sci USA 88:4756-4760.

Murphey RK, Matsumoto SG (1976) Experience modifies the plastic properties of identified neurons. Science 191:564-566.

Ouyang Y, Kantor D, Harris KM, Schuman EM, Kennedy MB (1997) Visualization of the distribution of autophosphorylated calcium/ calmodulin-dependent protein kinase II after tetanic stimulation in the CA1 region of the hippocampus. J Neurosci 17:5416-5427.

Reddy S, Jin P, Trimarchi JR, Caruccio P, Phillis RW, Murphey RK (1997) Mutant molecular motors disrupt neural circuits in Drosophila. J Neurobiol 33:711-723.

Robertson HM, Preston CR, Phillips RW, Johnson-Schlitz D, Benz WK, Engels WR (1988) A stable genomic source of P-element transposase in Drosophila melanogaster. Genetics 118:461-470.

SAS Institute (1989) SAS/STAT statistical software, version 6.12. Cary, NC: SAS Institute.

Shanbhag SR, Singh K, Singh RN (1992) Ultrastructure of the femoral chordotonal organs and their novel synaptic organization in the legs of Drosophila melanogaster meigene (Diptera: Drosophilidae). Int J Insect Morphol Embryol 21:311-322.

Silva AJ, Stevens CF, Tonegawa S, Wang Y (1992a) Deficient hippocampal long-term potentiation in $\alpha$-calcium/calmodulin kinase II mutant mice. Science 257:201-206.

Silva AJ, Paylor R, Wehner JM, Tonegawa ST (1992b) Impaired spatial learning in $\alpha$-calcium/calmodulin kinase II mutant mice. Science 257:206-211.

Stevens CF, Tonegawa S, Wang Y (1994) The role of calcium/calmodulin kinase II in three forms of synaptic plasticity. Curr Biol 4:687-693.

Sweeney ST, Broadie K, Keane J, Nieman H, O'Kane CJ (1995) Targeted expression of tetanus toxin light chain in Drosophila specifically eliminates synaptic transmission and causes behavioral defects. Neuron 14:341-351.

Trimarchi JR, Murphey RK (1997) The shaking- $\mathrm{B}^{2}$ mutation disrupts electrical synapses in a flight circuit in adult Drosophila. J Neurosci 17:4700-4710.

Trimarchi JR, Schneiderman AM (1993) Giant fiber activation of an intrinsic muscle in the mesothoracic leg of Drosophila melanogaster. J Exp Biol 177:149-167.

Trimarchi JR, Jin P, Murphey RK (1998) Controlling the motor neuron. In: Neuromuscular junction in Drosophila (Budnik V, Gramates S, eds). New York: Academic, in press.

Vandervorst P, Ghysen A (1980) Genetic control of sensory connection in Drosophila. Nature 286:65-67.

Wang J, Renger JJ, Griffith LC, Greenspan RJ, Wu C-F (1994) Concomitant alterations of physiological and developmental plasticity in Drosophila CaM kinase II-inhibited synapses. Neuron 13:1373-1384.

Wang Z, Palmer G, Griffith LC (1998) Regulation of Drosophila. $\mathrm{Ca}^{2+}$ / calmodulin-dependent protein kinase II by autophosphorylation analyzed by site-directed mutagenesis. J Neurochem 71:378-387.

Zou D-J, Cline HT (1996) Expression of constitutively active CaMKII in target tissue modifies presynaptic axon arbor growth. Neuron 16:529-539.

Zucker RS (1972) Crayfish escape behavior and central synapses. II. Physiological mechanisms underlying behavioral habituation. J Neurophysiol 35:621-637. 